\title{
An Efficient Transformation Technique for the Direct Recovering of the Antenna Far-Field Pattern from Near-Field Measurements Collected along a Helix
}

\author{
F. D’Agostino, F. Ferrara, C. Gennarelli", R. Guerriero and M. Migliozzi \\ D.I.In. - University of Salerno, via Ponte Don Melillo, 84084 Fisciano (Salerno), Italy
}

\begin{abstract}
A near-field to far-field transformation technique with helicoidal scanning for elongated antennas, which allows the evaluation of the antenna far-field pattern in any cut plane directly from a nonredundant number of near-field data without interpolating them, is developed in this paper. It is based on the nonredundant sampling representations of electromagnetic fields and employs a flexible source modelling suitable for long antennas to determine the number of helix turns. The number of near-field measurements on each turn is on the contrary dictated by the minimum cylinder rule, as in the classical cylindrical scanning, in order to reduce the computational burden and to simplify the scanning from the mechanical viewpoint. Some numerical and experimental results assessing the effectiveness of the proposed technique are reported.
\end{abstract}

Keywords: Antenna measurements, Direct near-field - far-field transformations, Helicoidal scanning, Nonredundant sampling representations of electromagnetic fields.

\section{INTRODUCTION}

Near-field - far-field (NF-FF) transformation techniques with helicoidal scanning, exploiting as suggested in [1] continuous and synchronized movements of the positioning systems of the probe and antenna under test (AUT), have been developed in [2-9] to significantly reduce the measurement time, which is currently very much greater than that needed to carry out the transformation. Apart from that in [2], they rely on the nonredundant sampling representations of electromagnetic (EM) fields $[10,11]$ and employ optimal sampling interpolation (OSI) formulas to efficiently recover the NF data required by the classical NFFF transformation with cylindrical scanning $[12,13]$ from the acquired nonredundant helicoidal ones. Besides the use of continuous movements, the drastic measurement time saving that characterizes the nonredundant $\mathrm{NF}-\mathrm{FF}$ transformations with helicoidal scanning [3-9] is due to the significantly reduced number of needed NF data, which can be further lowered if the surface chosen to enclose the AUT fits better its actual shape. This occurs, e.g., when considering elongated antennas which can be properly modelled as enclosed in a prolate ellipsoid [6-8] or in a cylinder ended in two half spheres (rounded cylinder) [8, 9], instead of the smallest sphere able to contain them [3-5]. Moreover, these effective antenna modellings allow the employment of scanning cylinders having radius smaller than one half the antenna maximum size, thus reducing the error due to the scanning area truncation. Indeed, also the

*Address correspondence to this author at the Dipartimento di Ingegneria Industriale (D.I.In.), University of Salerno, Via Ponte Don Melillo, 84084 Fisciano (SA), Italy; Tel. +39 089-964297; Fax +39 089-968730;

E-mail: cgennarelli@unisa.it probe uncompensated direct NF-FF transformation with helicoidal scanning [2], which employs a fast Fourier transform (FFT) based algorithm to evaluate the antenna far field, allows one to consider measurement cylinders with radius smaller than one half the antenna maximum size. However, such a technique does not exploit the nonredundant representations of EM fields and, accordingly, it needs a large amount of NF data. A direct NF-FF transformation with helicoidal scanning for volumetrical antennas, which allows the evaluation of the antenna far field in any cut plane directly from a nonredundant number of NF data without interpolating them, has been recently developed in [14]. Moreover, it presents the very interesting feature to remove the characteristic ripple caused by the discontinuity of the near field at the scanning zone edges. Unfortunately, this last transformation is not suitable for elongated antennas, as it makes use of the spherical source modelling. A direct helicoidal NF-FF transformation using a prolate ellipsoid to model an elongated AUT has been recently proposed $[15,16]$ in order to overcome the drawbacks related to the spherical source modelling.

The goal of this paper is to develop and validate both numerically and experimentally an even more effective direct NF-FF transformation with helicoidal scanning suitable for electrically long antennas and requiring a nonredundant number of NF measurements. Such a technique assumes the AUT as enclosed in a rounded cylinder (see Fig. 1) to determine the proper scanning helix and the number of its turns, whereas the amount of NF data required on each turn is fixed according to the minimum cylinder rule, as in standard cylindrical scanning $[12,13]$. The choice of a rounded cylinder instead of a prolate ellipsoid as AUT modelling allows a further reduction of the number of NF data and, as a consequence, of the 


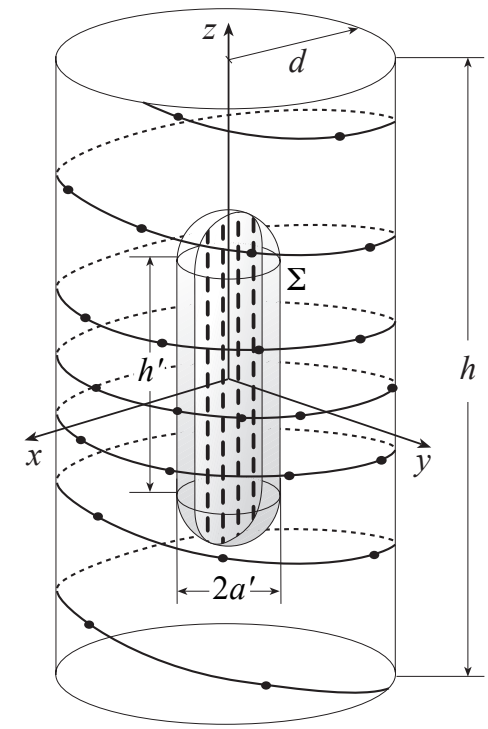

Fig. (1). Helicoidal scanning for electrically long antennas.

Fig. (2). Relevant to a generatrix.

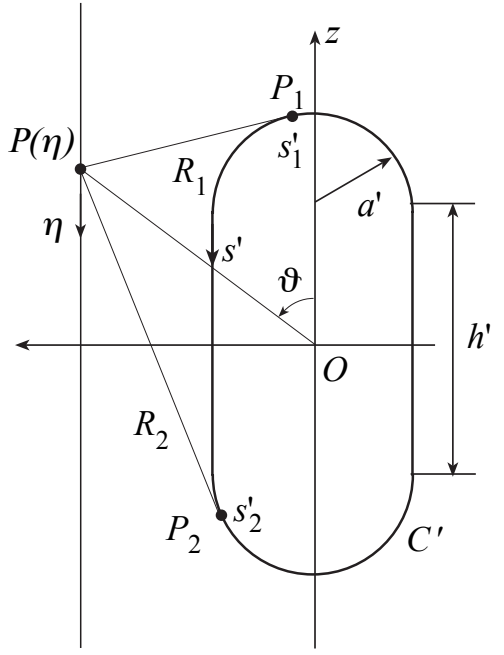

measurement time. As a matter of fact, such a flexible modelling allows one to fit better the shape of a lot of actual antennas by properly setting its geometric parameters.

\section{NONREDUNDANT SAMPLING REPRESENTATION OF THE VOLTAGE}

Let us consider an elongated AUT enclosed in a rounded cylinder $\Sigma$ of parameters $h^{\prime}$ and $a^{\prime}$ and a nondirective probe scanning a proper helix lying on a cylinder of radius $d$ in the antenna NF region (Fig. 1). In the following, the spherical coordinate system $(r, \vartheta, \varphi)$ or the cylindrical one $(\rho, \varphi, z)$ are used to denote an observation point $P$. Since the voltage $V$ measured by such a probe has the same effective spatial bandwidth of the field [17], the nonredundant representations of EM fields [10] can be applied to it. Accordingly, when dealing with the representation of $V$ on an observation curve $C$, it is convenient to adopt a proper analytical parameterization $\underline{r}=\underline{r}(\eta)$ for describing $C$ and to introduce the "reduced voltage"

$$
\tilde{V}(\eta)=V(\eta) \mathrm{e}^{\mathrm{j} \psi(\eta)}
$$

where $V$ is the voltage $V_{1}$ or $V_{2}$ measured by the probe or by the rotated probe and $\psi(\eta)$ is a proper phase function. The error, occurring when $\tilde{V}(\eta)$ is approximated by a bandlimited function, is negligible as the bandwidth exceeds a critical value $W_{\eta}$ [10], so that it can be controlled by choosing a bandwidth equal to $\chi^{\prime} W_{\eta}$, where $\chi^{\prime}$ is a bandwidth enlargement factor slightly greater than unity for an electrically large antenna [10].

When the curve $C$ is a cylinder generatrix, the bandwidth $W_{\eta}$, the parameterization $\eta$, and the corresponding phase function $\psi$ are $[9,10]$ :

$$
\begin{aligned}
& W_{\eta}=\beta \ell^{\prime} / 2 \pi ; \eta=\left(\pi / \ell^{\prime}\right)\left[R_{1}-R_{2}+s_{1}^{\prime}+s_{2}^{\prime}\right] \\
& \psi=(\beta / 2)\left[R_{1}+R_{2}+s_{1}^{\prime}-s_{2}^{\prime}\right]
\end{aligned}
$$

where $\beta$ is the wave number, $\ell^{\prime}$ is the length of $C^{\prime}$ (intersection curve between the meridian plane passing through $P$ and $\Sigma), R_{1,2}$ are the distances from $P(d, \varphi, z)$ to the tangency points $P_{1,2}$ on $C^{\prime}$, and $s_{1,2}^{\prime}$ are their arclength coordinates (see Fig. 2). As shown in [9], it results:

$$
\ell^{\prime}=2\left(h^{\prime}+\pi a^{\prime}\right) ; R_{1,2}=\sqrt{\left(z \mp h^{\prime} / 2\right)^{2}+d^{2}-a^{\prime 2}}
$$




$$
\begin{aligned}
& s_{1}^{\prime}=a^{\prime} \sin ^{-1}\left(\frac{a^{\prime} d+R_{1}\left(\left(h^{\prime} / 2\right)-z\right)}{R_{1}^{2}+a^{\prime 2}}\right) \\
& s_{2}^{\prime}=h^{\prime}+a^{\prime}\left[\pi-\sin ^{-1}\left(\frac{a^{\prime} d+R_{2}\left(\left(h^{\prime} / 2\right)+z\right)}{R_{2}^{2}+a^{\prime 2}}\right)\right]
\end{aligned}
$$

According to $[8,9]$, a two-dimensional OSI expansion to reconstruct the probe voltage from a non redundant number of its samples acquired along a helix is obtained: i) by determining the helix such that its step is equal to sample spacing needed for the voltage interpolation along a generatrix; ii) by developing a non redundant sampling representation of the voltage on the helix.

Accordingly, the helix is got by projecting on the cylinder a proper spiral wrapping the rounded cylinder that models the AUT. The helix step is equal to the sample spacing $\Delta \eta=2 \pi /\left(2 \mathrm{~N}^{\prime \prime}+1\right)$ needed for the voltage interpolation along a generatrix. Note that $N^{\prime \prime}=\operatorname{Int}\left(\chi N^{\prime}\right)+1$, where $\operatorname{Int}(x)$ denotes the integer part of $x, \chi>1$ is an oversampling factor that controls the truncation error [10], and $N^{\prime}=$ Int $\left(\chi^{\prime} W_{\eta}\right)+1$. The projection is obtained via the curves at $\eta=$ const $[8,9]$. Thus, the parametric equations of the helix, when imposing its passage through a point $Q_{0}$ of the generatrix at $\varphi=0$, are:

$$
\left\{\begin{array}{l}
x=d \cos \left(\phi-\phi_{s}\right) \\
y=d \sin \left(\phi-\phi_{s}\right) \\
z=d \cot [\vartheta(\eta)]
\end{array}\right.
$$

where in $\phi$ is the angular parameter that describes the helix, $\phi_{s}$ is the value of $\phi$ at $Q_{0}$, and $\eta=k \phi$, with $k=\Delta \eta / 2 \pi=1 /\left(2 N^{\prime \prime}+1\right)$.

The nonredundant representation on the helix is got by choosing the optimal parameter $\xi$ describing it equal to $\beta / W_{\xi}$ times the arclength of the projecting point on the spiral wrapping the rounded cylinder and the related phase function $\gamma$ coincident with $\psi$. The bandwidth $W_{\xi}$ is chosen equal to $\beta / \pi$ times the length of the spiral wrapping $\Sigma$ from pole to pole [8].

According to the above results, the reduced voltage at the point $P(d, \varphi, z)$ can be recovered via the OSI expansion along the generatrix [9]:

$$
\tilde{V}(\eta(z), \varphi)=\sum_{n=n_{0}-q+1}^{n_{0}+q} \tilde{V}\left(\eta_{n}\right) \Omega_{N}\left(\eta-\eta_{n}\right) D_{N^{\prime \prime}}\left(\eta-\eta_{n}\right)
$$

where $2 q$ is the number of retained samples

$$
\begin{aligned}
& \tilde{V}\left(\eta_{n}\right), n_{0}=\operatorname{Int}\left[\left(\eta-\eta_{0}\right) / \Delta \eta\right], \\
& \eta_{n}=\eta_{n}(\varphi)=\eta\left(\phi_{s}\right)+k \varphi+n \Delta \eta=\eta_{0}+n \Delta \eta
\end{aligned}
$$

and

$$
\begin{aligned}
& D_{N^{\prime \prime}}(\eta)=\frac{\sin \left[\left(2 N^{\prime \prime}+1\right) \eta / 2\right]}{\left(2 N^{\prime \prime}+1\right) \sin (\eta / 2)} \\
& \Omega_{N}(\eta)=\frac{T_{N}\left[2 \cos ^{2}(\eta / 2) / \cos ^{2}(\bar{\eta} / 2)-1\right]}{T_{N}\left[2 / \cos ^{2}(\bar{\eta} / 2)-1\right]}
\end{aligned}
$$

are the Dirichlet and Tschebyscheff sampling functions [9, 10], $T_{N}($.$) being the Tschebyscheff polynomial of degree$ $\mathrm{N}=N^{\prime \prime}-N^{\prime}$ and $\bar{\eta}=q \Delta \eta$.

The samples $\tilde{V}\left(\eta_{n}\right)$ are recovered [9] via a similar OSI expansion along the helix:

$$
\tilde{V}\left(\xi\left(\eta_{n}\right)\right)=\sum_{m=m_{0}-p+1}^{m_{0}+p} \tilde{V}\left(\xi_{m}\right) \Omega_{M}\left(\xi-\xi_{m}\right) D_{M^{\prime \prime}}\left(\xi-\xi_{m}\right)
$$

where $m_{0}=\operatorname{Int}\left[\left(\xi-\xi_{S}\right) / \Delta \xi\right]$ and

$$
\xi_{m}=\xi\left(\phi_{S}\right)+m \Delta \xi=\xi_{S}+2 \pi m /\left(2 M^{\prime \prime}+1\right)
$$

with $M^{\prime \prime}=\operatorname{Int}\left(\chi M^{\prime}\right)+1$ and $M^{\prime}=\operatorname{Int}\left(\chi^{\prime} W_{\xi}\right)+1$. The other symbols have the same meaning as in (8).

Expansions (8) and (12) allow the reconstruction of the probe voltage at any point on the scanning cylinder from the acquired nonredundant helicoidal samples so that they can be properly applied to recover the NF data required by the classical cylindrical NF-FF transformation [12] or [13]. However, the proposed direct NF-FF transformation avoids such an interpolation step, as it will be shown in the next section.

\section{THE DIRECT NF-FF TRANSFORMATION TECHNIQUE}

According to [12], the cylindrical wave expansion coefficients $a_{v}$ and $b_{v}$ of the AUT field are related to: i) the two-dimensional Fourier transforms

$$
I_{v}^{1,2}(\tau)=\int_{-\infty}^{\infty} \int_{0}^{2 \pi} V^{1,2}(\varphi, z) \mathrm{e}^{-\mathrm{j} v \varphi} \mathrm{e}^{\mathrm{j} \tau z} \mathrm{~d} \varphi \mathrm{d} z
$$

of the probe voltage for two sets of measurements (the probe is rotated by $90^{\circ}$ in the second set); ii) the wave expansion coefficients $\left(c_{m}, d_{m}\right)$ and $\left(c^{\prime}{ }_{m}, d^{\prime}{ }_{m}\right)$ of the field radiated by the probe and the rotated probe, when used as transmitting antennas. In particular [12],

$$
\begin{aligned}
& a_{v}(\tau)=\frac{\beta^{2}}{\Lambda^{2} \Delta_{v}(\tau)}\left[I_{v}^{1}(\tau) \sum_{m=-\infty}^{\infty} d_{m}^{\prime}(-\tau) H_{v+m}^{(2)}(\Lambda d)+\right. \\
& \left.-I_{v}^{2}(\tau) \sum_{m=-\infty}^{\infty} d_{m}(-\tau) H_{v+m}^{(2)}(\Lambda d)\right] \\
& b_{v}(\tau)=\frac{\beta^{2}}{\Lambda^{2} \Delta_{v}(\tau)}\left[I_{v}^{2}(\tau) \sum_{m=-\infty}^{\infty} c_{m}(-\tau) H_{v+m}^{(2)}(\Lambda d)+\right. \\
& \left.-I_{v}^{1}(\tau) \sum_{m=-\infty}^{\infty} c_{m}^{\prime}(-\tau) H_{v+m}^{(2)}(\Lambda d)\right]
\end{aligned}
$$

where in

$\Delta_{v}(\tau)=\sum_{m=-\infty}^{\infty} c_{m}(-\tau) H_{v+m}^{(2)}(\Lambda d) \sum_{m=-\infty}^{\infty} d_{m}^{\prime}(-\tau) H_{v+m}^{(2)}(\Lambda d)+$ 


$$
-\sum_{m=-\infty}^{\infty} c_{m}^{\prime}(-\tau) H_{v+m}^{(2)}(\Lambda d) \sum_{m=-\infty}^{\infty} d_{m}(-\tau) H_{v+m}^{(2)}(\Lambda d)
$$

where $\Lambda=\left(\beta^{2}-\tau^{2}\right)^{1 / 2}$, and $H_{v}^{(2)}(\cdot)$ is the Hankel function of second kind and order $v$.

In the classical approach, the Fourier transforms (14) of the probe and rotated probe voltages are efficiently evaluated via the FFT algorithm and the sample spacings of the NF data are:

$$
\Delta z \leq \lambda / 2 ; \Delta \varphi \leq \pi /\left(\beta \rho^{\prime}\right)=\lambda /\left(2 \rho^{\prime}\right)
$$

where $\rho^{\prime}$ is the radius of the smallest cylinder enclosing the AUT (minimum cylinder rule) and $\lambda$ the wave length.

Once the modal coefficients have been determined, the FF components of the electric field

$$
\begin{aligned}
& E_{\vartheta}(r, \vartheta, \varphi)=-\mathrm{j} 2 \beta \frac{\mathrm{e}^{-\mathrm{j} \beta r}}{r} \sin \vartheta \sum_{v=-\infty}^{\infty} \mathrm{j}^{v} b_{v}(\beta \cos \vartheta) \mathrm{e}^{\mathrm{j} v \varphi} \\
& E_{\varphi}(r, \vartheta, \varphi)=-2 \beta \frac{\mathrm{e}^{-\mathrm{j} \beta r}}{r} \sin \vartheta \sum_{\nu=-\infty}^{\infty} \mathrm{j}^{v} a_{v}(\beta \cos \vartheta) \mathrm{e}^{\mathrm{j} v \varphi}
\end{aligned}
$$

in the spherical coordinate system $(r, \vartheta, \varphi)$ can be efficiently evaluated by performing the summations via the FFT algorithm.

An efficient probe compensated NF-FF transformation to evaluate the antenna far field directly from the nonredundant helicoidal NF data is now proposed. To this end, by taking into account the OSI expansion (8) and relation (1), the Fourier transforms (14) are rewritten in the form:

$$
\begin{aligned}
& I_{v}^{1,2}(\tau)=\sum_{n \in N_{r}} \int_{0}^{2 \pi}\left\{\tilde{V}^{1,2}\left(\eta_{n}\right) \mathrm{e}^{-\mathrm{j} v \varphi} .\right. \\
& \left.\cdot \int_{-\infty}^{\infty} D_{N^{\prime \prime}}\left(\eta(z)-\eta_{n}\right) Q\left(\eta(z)-\eta_{n}\right) \mathrm{e}^{-\mathrm{j} \psi(z)} \mathrm{e}^{\mathrm{j} \tau z} \mathrm{~d} z\right\} \mathrm{d} \varphi
\end{aligned}
$$

where, according to (9), $\tilde{V}^{1,2}\left(\eta_{n}\right)$ are the reduced samples at the intersection points between the generatrix at $\varphi$ and the helix, $N_{r}$ is the set of indexes of all used helix turns, and the function $Q$ coincides with the function $\Omega_{N}$, when $\left|\eta(\mathrm{z})-\eta_{n}\right| \leq$ $q \Delta \eta$, and is equal to 0 , otherwise.

For any fixed $\varphi$, the integration over $z$, gives:

$$
G_{n \tau}(\varphi)=\int_{z_{i}}^{z_{f}} D_{N^{\prime \prime}}\left(\eta(z)-\eta_{n}\right) \Omega_{N}\left(\eta(z)-\eta_{n}\right) \mathrm{e}^{-\mathrm{j} \psi(z)} \mathrm{e}^{\mathrm{j} \tau z} \mathrm{~d} z
$$

where $z_{i}=z\left(\eta_{n}+q \Delta \eta\right)$ and $z_{f}=z\left(\eta_{n}-q \Delta \eta\right)$. Thus relation (21) can be rewritten as

$$
I_{v}^{1,2}(\tau)=\sum_{n \in N_{r}} \int_{0}^{2 \pi} \tilde{V}^{1,2}\left(\eta_{n}\right) G_{n \tau}(\varphi) \mathrm{e}^{-\mathrm{j} v \varphi} \mathrm{d} \varphi
$$

It can be easily recognized that the integration over $\varphi$ in this last relation can be efficiently performed via the FFT algorithm, if the number of the samples on each helix turn is always equal to the smallest integer $M_{H}$, product of powers of 2, 3 and 5 equal or greater than $2\left[\operatorname{Int}\left(\chi^{\prime} \beta a^{\prime}\right)+1\right]$. Note that, in such a way, the samples lying on the helix at $\varphi_{m}=m \Delta \varphi=$ $2 \pi m / M_{H}$ with $m=0, \ldots, M_{H}-1$ are all aligned and, accordingly, it results:

$$
\begin{aligned}
& \int_{0}^{2 \pi} \tilde{V}^{1,2}\left(\eta_{n}\right) G_{n \tau}(\varphi) \mathrm{e}^{-\mathrm{j} v \varphi} \mathrm{d} \varphi= \\
& \frac{2 \pi}{M_{H}} \sum_{m=0}^{M_{H}-1} \tilde{V}^{1,2}\left(\eta_{m, n}\right) G_{n \tau}\left(\varphi_{m}\right) \mathrm{e}^{-\mathrm{j} \frac{2 \pi m v}{M_{H}}}
\end{aligned}
$$

where $\eta_{m . n}=\eta_{n}\left(\varphi_{m}\right)=\eta\left(\phi_{s}\right)+k \varphi_{m}+n \Delta \eta$. The summation in (24) can be, obviously, carried out via FFT. Moreover, it is worthy to stress that the $G_{n \tau}\left(\varphi_{m}\right)$ 's can be calculated, once and for all, for given sets of antennas, since they depend only on the measurement cylinder radius and on the parameters of the AUT modelling.

By summing up, the antenna far field can be determined by executing the following steps: i) the samples of $V^{1}$ and $V^{2}$, acquired at constant angular step $\Delta \varphi$ along the helix, are multiplied by the phase factor $\mathrm{e}^{\mathrm{j} \psi}$; ii) for each required value of $\vartheta$, fixing the corresponding value of $\tau$ via the relation $\tau=\beta \cos \vartheta$, the $G_{n \tau}$ values are evaluated or read, if already calculated; iii) for each helix turn, specified by the index $n$, the FFT of the sequence $\tilde{V}^{1,2}\left(\eta_{m, n}\right) G_{n \tau}\left(\varphi_{m}\right)$ is computed; iv) then the Fourier transforms $I_{v}^{1,2}(\tau)$ of the probe voltages are determined by performing the summations over $n \in N_{r}$ and the corresponding values of the modal coefficients $a_{v}(\tau)$ and $b_{v}(\tau)$ are evaluated; $\left.\mathrm{v}\right)$ at last, the FF spherical components of the electric field at the considered angle $\vartheta$ are determined by evaluating (19) and (20) via the FFT algorithm.

In order to reduce the computational effort, it is convenient to employ this procedure to evaluate only the FF samples required for reconstructing the antenna pattern via the OSI expansion in [18], properly modified to deal with an even number of samples both along the parallels and the meridians:

$$
\begin{gathered}
F_{\vartheta, \varphi}(\vartheta(\eta), \varphi)=\frac{2 N_{F}^{\prime \prime}-1}{2 N_{F}^{\prime \prime}} \sum_{n=n_{0}-q+1}^{n_{0}+q}\left\{\Omega_{N_{F}}\left(\eta-\eta_{n}\right) \cdot\right. \\
\cdot D_{N_{F}^{\prime \prime}-1}\left(\eta-\eta_{n}\right) \frac{2 M_{n}^{\prime \prime}-1}{2 M_{n}^{\prime \prime}} \sum_{m=m_{0}-p+1}^{m_{0}+p} F_{\vartheta, \varphi}\left(\eta_{n}, \varphi_{m, n}\right) \cdot \\
\left.\cdot \Omega_{M_{n}}\left(\varphi-\varphi_{m, n}\right) D_{M_{n}^{\prime \prime}-1}\left(\varphi-\varphi_{m, n}\right)\right\}
\end{gathered}
$$

where in $n_{0}=\operatorname{Int}(\eta / \Delta \eta)$ and $m_{0}=\operatorname{Int}\left(\varphi / \Delta \varphi_{n}\right)$ are the indexes of the sample nearest (on the left) to the output point, and 


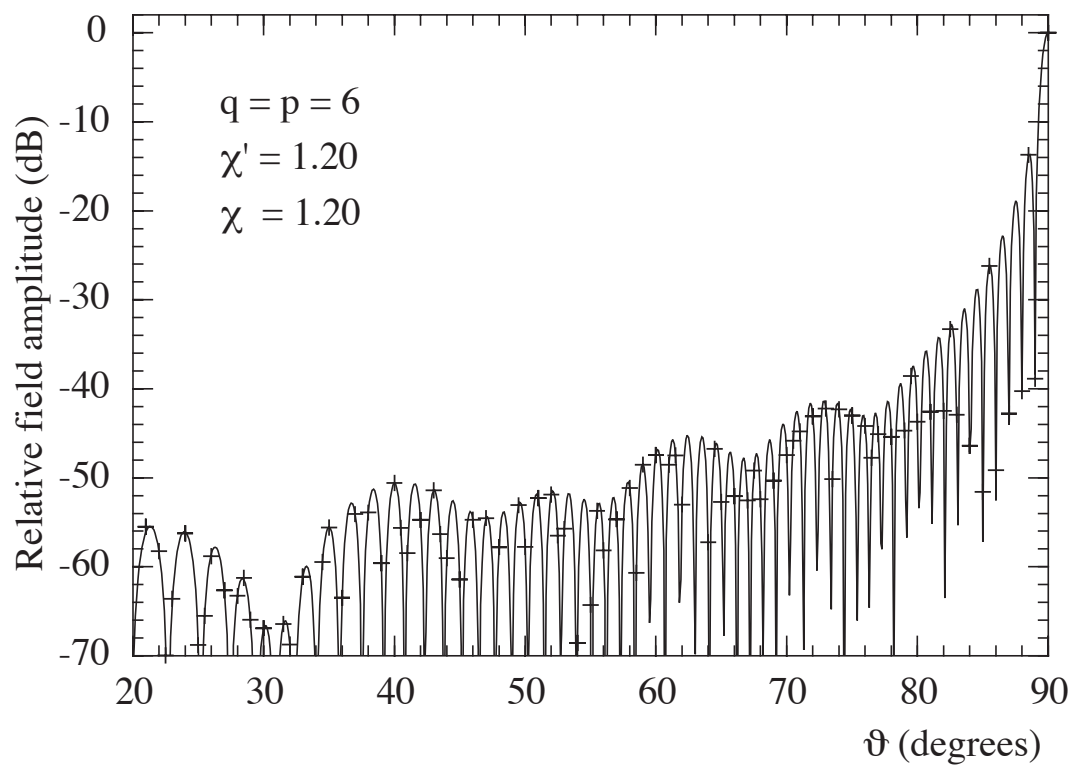

Fig. (3). E-plane pattern. Solid line: exact. Crosses: recovered via the direct NF-FF transformation with helicoidal scanning.

$$
\begin{aligned}
& F_{\vartheta, \varphi}(\vartheta, \varphi)=r \mathrm{e}^{\mathrm{j} \beta r} E_{\vartheta, \varphi}(r, \vartheta, \varphi) \\
& \eta_{n}=n \Delta \eta=n \pi / N_{F}^{\prime \prime} ; \varphi_{m, n}=m \Delta \varphi_{n}=m \pi / M_{n}^{\prime \prime} \\
& N_{F}^{\prime \prime}=2\left[\operatorname{Int}\left(\chi N^{\prime} / 2\right)+1\right] ; N_{F}=N_{F}^{\prime \prime}-N^{\prime} \\
& M_{n}^{\prime \prime}=2^{i} \geq \operatorname{Int}\left(\chi M_{n}^{\prime}\right)+1 ; M_{n}^{\prime}=\operatorname{Int}\left[\chi^{*} W_{\varphi}\left(\eta_{n}\right)\right]+1 \\
& W_{\varphi}\left(\eta_{n}\right)=\beta a^{\prime} \sin \left(\vartheta\left(\eta_{n}\right)\right) ; M_{n}=M_{n}^{\prime \prime}-M_{n}^{\prime} \\
& \chi^{*}=1+\left(\chi^{\prime}-1\right)\left[\sin \vartheta\left(\eta_{n}\right)\right]^{-2 / 3}
\end{aligned}
$$

It must be stressed that there is no need to extract the phase factor $\mathrm{e}^{-\mathrm{j} \Psi(\eta)}$ from the far-field expression, since it is constant on the far-field sphere. Moreover, in the considered far-field representation, the expression of the optimal parameter $\eta$ becomes simpler [18]:

$$
\eta=\left(\pi / \ell^{\prime}\right)\left[(1-\cos \vartheta) h^{\prime}+2 a^{\prime} \vartheta\right]
$$

It is worth noting that the use of an OSI expansion tailored for an even number of samples along the parallels is due to the employment of an efficient power of two FFT algorithm for computing the FF components of the electric field (19) and (20). Moreover, $N_{F}^{\prime \prime}$ has been chosen according to (28) in order to have FF samples on the equator.

It can be useful to point out that, by applying the OSI expansion (25), it is possible to reconstruct the antenna far field in any cut plane at $\varphi=$ constant and not only in those attainable by performing the evaluation of (19) and (20) via FFT. At last, it must be stressed that, as shown in [14], the proposed direct NF-FF transformation with helicoidal scanning exhibits the very interesting feature to eliminate the ripple due to the near field discontinuity at the edges of the scanning zone. This is due to the different method employed to evaluate $I_{v}^{1,2}$. As a matter of fact, in the standard transformation technique, they are computed via FFT by taking into account only the NF data falling in the scanning area so that the integration over $z$ is truncated to the measurement cylinder height. Whereas, in the proposed approach, the effect of each NF sample is considered (see eq. (22)) in the range $\left[z_{i}, z_{f}\right]$, so that the peripheral samples affect the evaluation even far from the scanning area boundaries. Thus, the effect due to the field discontinuity at the scanning zone edges is intrinsically eliminated.

\section{NUMERICAL SIMULATIONS}

The following simulation refers to a uniform planar array of $\lambda / 2$ spaced elementary Huygens sources, polarized along the $z$ axis and covering a zone in the plane $y=0$, formed by a rectangle ended in two half-circles. The sizes of the rectangle are: $2 a^{\prime}=14 \lambda$ and $h^{\prime}=46 \lambda$. The helix wraps a cylinder with radius $d=14 \lambda$ and height $h=160 \lambda$. An open-ended WR-90 rectangular waveguide, at the frequency of $10 \mathrm{GHz}$, is chosen as measurement probe.

The reconstruction of the antenna far-field pattern in the principal planes obtained by employing the proposed direct NF-FF transformation with helicoidal scanning is shown in Figs. (3 and 4). As can be seen, in both the planes, the exact and reconstructed patterns are indistinguishable, thus assessing the effectiveness of the developed transformation technique. In order to show the previously claimed capability of the technique to accurately recover the antenna far field in any cut plane, the reconstructions of the amplitudes of both the far-field components in the plane at $\varphi=60^{\circ}$ are reported in Figs. (5 and 6).

It worth noting that the number of employed samples is 20213 , significantly less than that (34 668) required by the standard cylindrical NF-FF transformation $[12,13]$ and by the half-wavelength helicoidal scanning [2], to cover the same measurement area. 


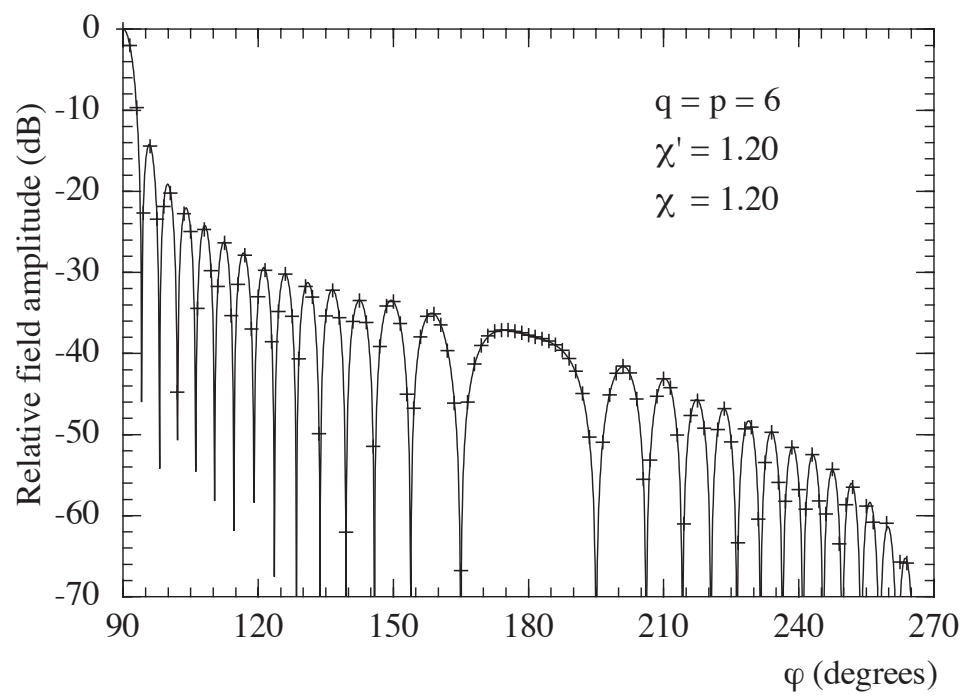

Fig. (4). H-plane pattern. Solid line: exact. Crosses: recovered via the direct NF-FF transformation with helicoidal scanning.

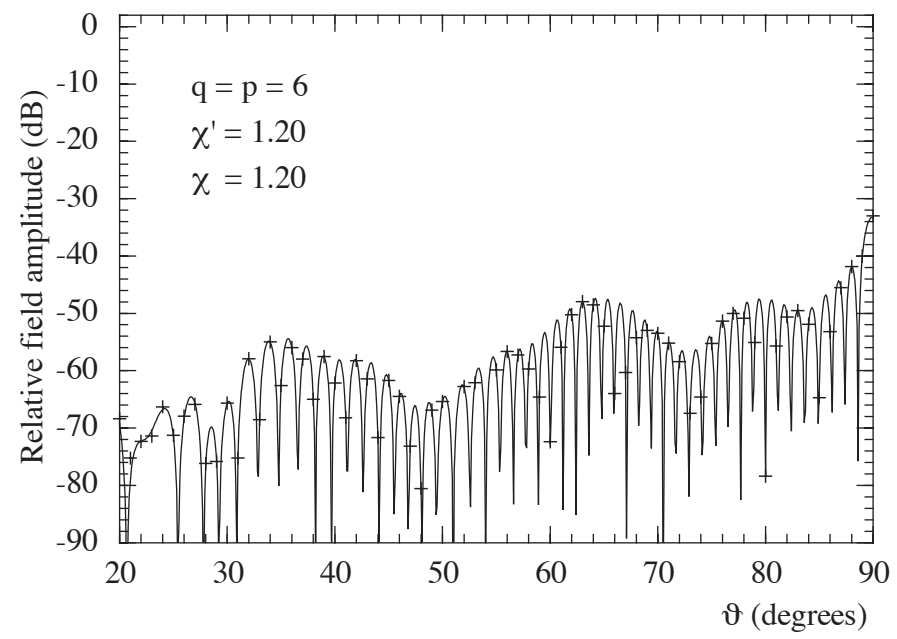

Fig. (5). Amplitude of the far-field $\vartheta$-component at $\varphi=60^{\circ}$. Solid line: exact. Crosses: recovered via the direct NF-FF transformation with helicoidal scanning.

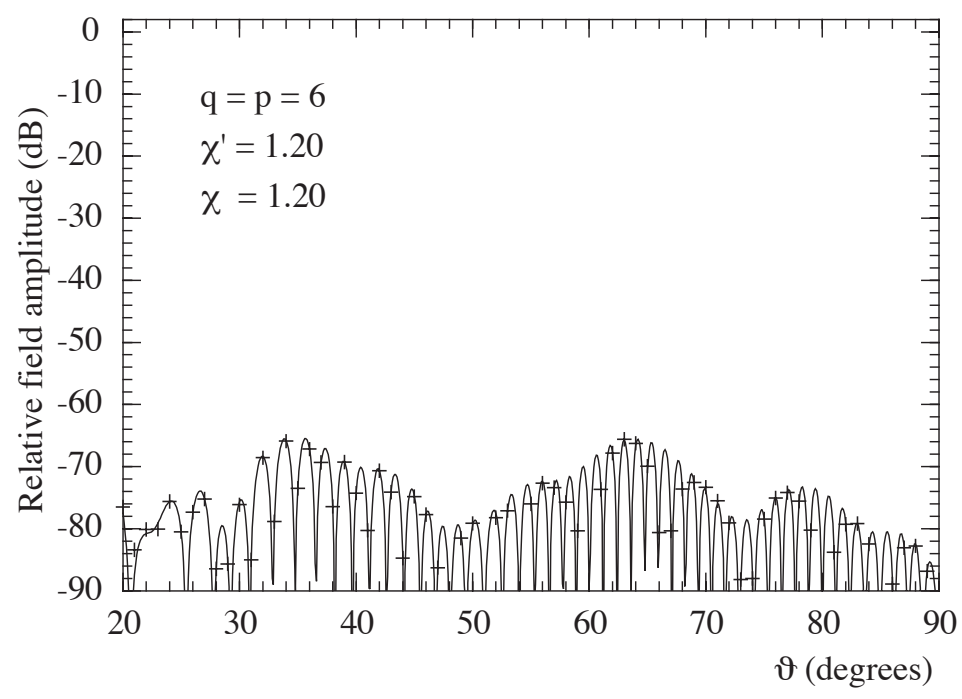

Fig. (6). Amplitude of the far-field $\varphi$-component at $\varphi=60^{\circ}$. Solid line: exact. Crosses: recovered via the direct NF-FF transformation with helicoidal scanning. 


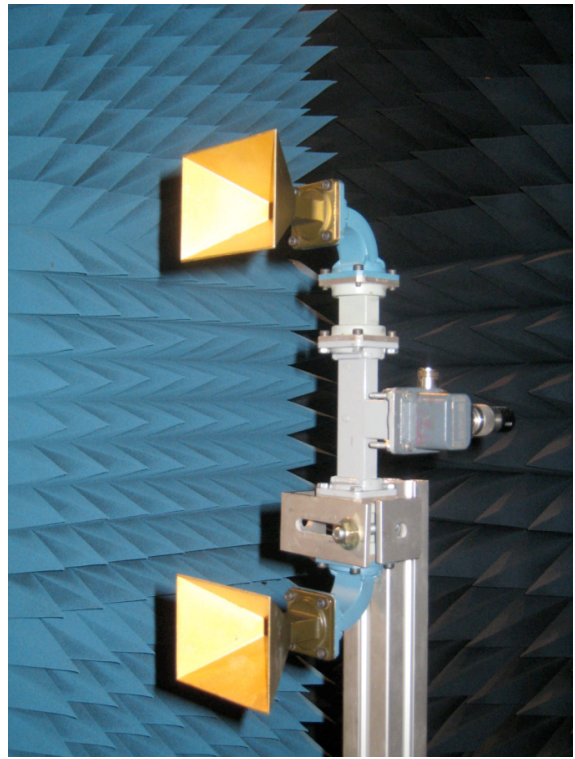

Fig. (7). Photo of the E-plane monopulse antenna.

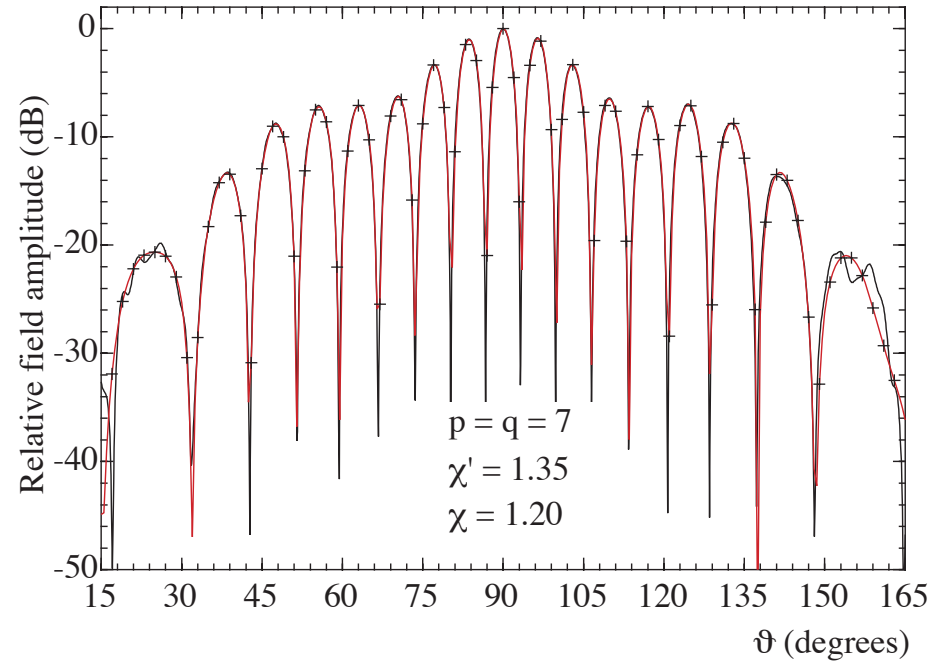

Fig. (8). E-plane pattern of the AUT 1. Solid line: reference. Red solid line with crosses: recovered via the direct NF-FF transformation with helicoidal scanning.

\section{EXPERIMENTAL TESTS}

The experimental tests on the proposed direct helicoidal NF-FF transformation have been carried out in the anechoic chamber of the Antenna Characterization Lab of the University of Salerno, where an advanced cylindrical NF scanning system supplied by MI-Technologies is available. The NF data have been acquired by an open-ended WR-90 rectangular wave guide along a helix wrapping a cylinder with radius $d=19.6 \mathrm{~cm}$ and height $h=240 \mathrm{~cm}$. A vectorial network analyzer has been employed to perform the amplitude and phase measurements.

Two different monopulse antennas operating at $10 \mathrm{GHz}$ in the sum mode have been employed in the experimental tests. They have been obtained by properly assembling two pyramidal horns $(8.9 \times 6.8 \mathrm{~cm}$ sized $)$, a hybrid tee, and two straight and two curved rectangular waveguides. The former (AUT 1) is an E-plane monopulse antenna (see Fig. 7), wherein the distance between the centers of the pyramidal horns is $26.5 \mathrm{~cm}$. The latter (AUT 2), on the contrary, is a H- plane monopulse antenna with a distance between the horns centers of $26 \mathrm{~cm}$. The apertures of both antennas are located in the plane $y=0$ of the adopted reference system. A rounded cylinder of parameters $h^{\prime}=33.3 \mathrm{~cm}$ and $a^{\prime}=4.95$ $\mathrm{cm}$ has been adopted to model the former antenna, whereas the latter has been considered as enclosed in a rounded cylinder with $h^{\prime}=36 \mathrm{~cm}$ and $a^{\prime}=4.5 \mathrm{~cm}$.

The direct NF-FF transformation incorporates the probe characterization. Accordingly, in a previous paper [19], we have characterized the used probe according to [20] as done in the software package MI-3000 implementing the standard probe compensated NF-FF transformation [13], and verified that practically identical results are obtained when the same NF data are transformed by using the MI-Technologies software and our version of the probe compensated NF-FF transformation technique [12].

In the next Figs. (8 to 11), the FF patterns in the principal planes $\mathrm{E}$ and $\mathrm{H}$ reconstructed via the direct $\mathrm{NF}-\mathrm{FF}$ transformation are compared with those (references) 


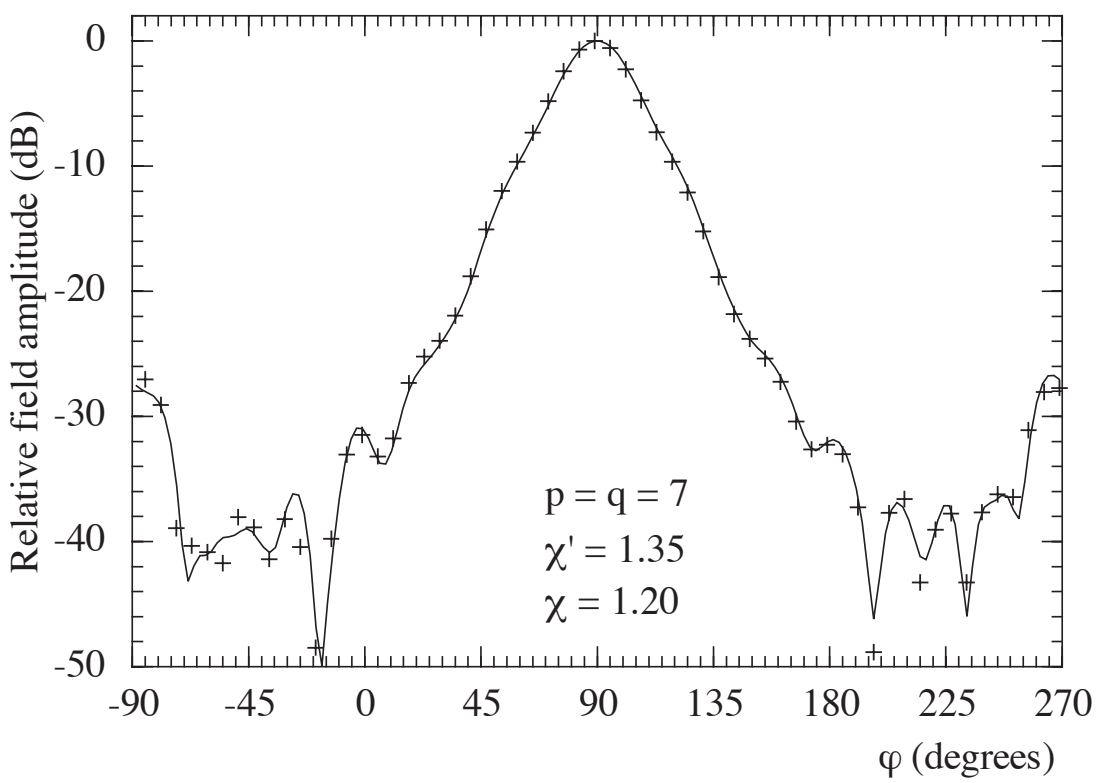

Fig. (9). H-plane pattern of the AUT 1. Solid line: reference. Crosses: recovered via the direct NF-FF transformation with helicoidal scanning.

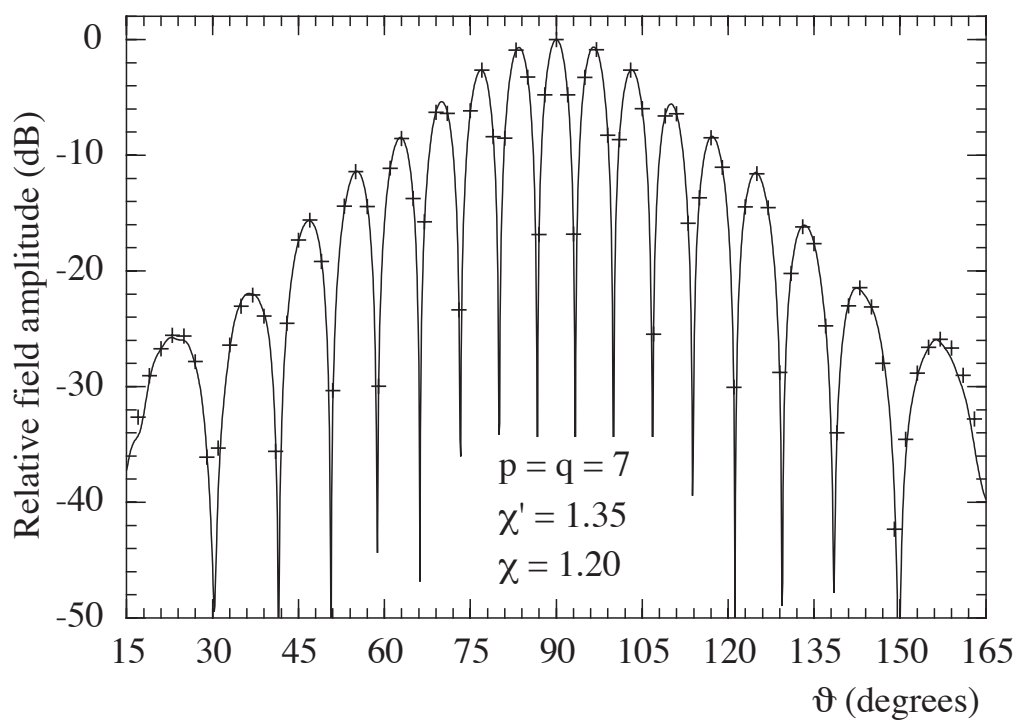

Fig. (10). H-plane pattern of the AUT 2. Solid line: reference. Crosses: recovered via the direct NF-FF transformation with helicoidal scanning.

obtained by using the MI software from NF data measured on the classical cylindrical grid. In particular, Figs. (8 and 9) are relevant to the AUT 1, whereas Figs. (10 and 11) refer to the AUT 2. As can be seen, a very good agreement results, thus further assessing the effectiveness of the proposed technique. It is worth noting that the ripple caused by the discontinuity of the near field at the edges of the scanning surface, clearly visible in the E-plane pattern obtained from the NF data measured on the classical cylindrical grid when considering the AUT 1 (see Fig. 8), is thoroughly eliminated in the FF pattern reconstructed by using the direct $\mathrm{NF}-\mathrm{FF}$ transformation. Such a ripple is not visible in Fig. (10), since the near field level at the edges of the scanning area when considering the latter antenna is about $20 \mathrm{~dB}$ lower than for the former one.

It can be interesting to compare the number of NF data needed by the here proposed direct helicoidal NF-FF transformation with those required by the other transformation techniques (see Table 1).

As regards the time needed to acquire the NF data, the helicoidal scanning technique proposed in [2] is certainly quicker than the classical cylindrical one $[12,13]$, since the acquisition is carried out by means of continuous and synchronized movements of the positioning systems. In light of the above consideration, it is enough to compare the here proposed technique only with the one in [2]. In such a case, it can be easily realized that the measurement times are directly proportional to the numbers of required samples.

\section{CONCLUSIONS}

A direct NF-FF transformation with helicoidal scanning for electrically long antennas has been developed and assessed both by means of numerical simulations and experi- 


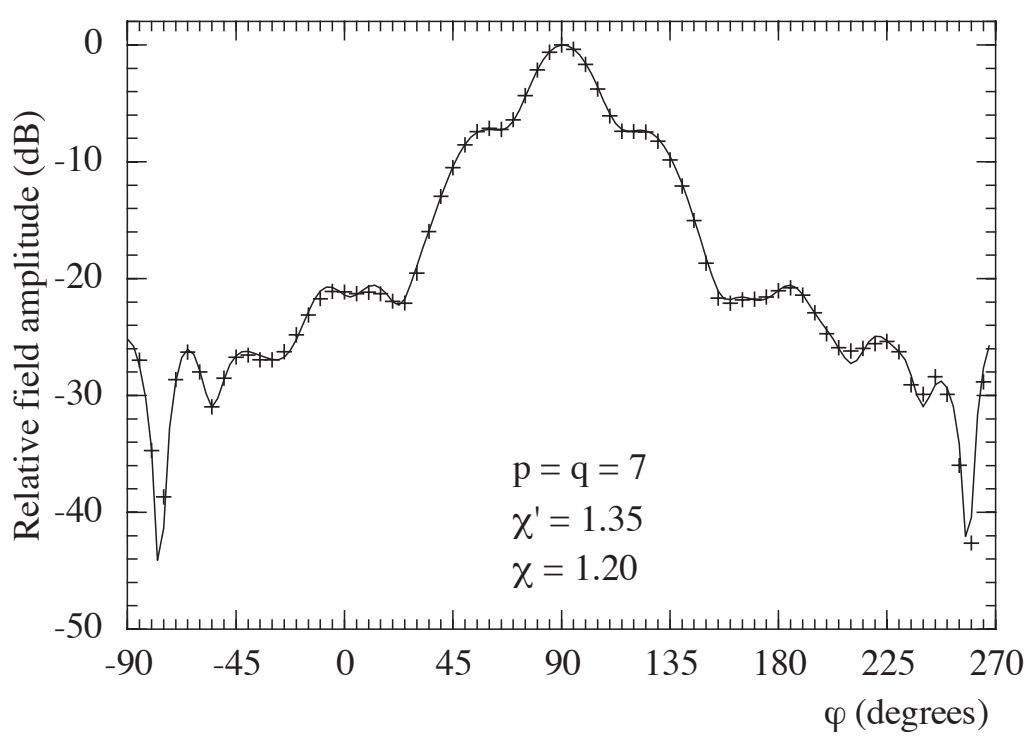

Fig. (11). E-plane pattern of the AUT 2. Solid line: reference. Crosses: recovered via the direct NF-FF transformation with helicoidal scanning.

Table 1. Data Required by the Different NF-FF Transformation Techniques

\begin{tabular}{|c|c|c|}
\hline NF-FF Transformation Technique & AUT 1 $\boldsymbol{N}_{\text {data }}$ & AUT 2 $\boldsymbol{N}_{\boldsymbol{d a t a}}$ \\
\hline \hline Standard cylindrical scanning [12, 13] or half-wavelength helicoidal scanning [2] & 6440 & 5796 \\
\hline Direct helicoidal scanning using a prolate ellipsoidal modelling [15] & 2202 & 1986 \\
\hline Here proposed approach & 2048 & 1954 \\
\hline
\end{tabular}

mental tests. It makes possible the evaluation of the antenna far field in any cut plane directly from the acquired helicoidal NF data without interpolating them. Moreover it exhibits the appealing characteristic to eliminate the ripple due to the discontinuity of the near field at the edges of the scanning area. The number of helix turns is determined by the rounded cylinder AUT modelling, whereas the number of data on each of them is fixed by the minimum cylinder rule, as in the standard cylindrical scanning, thus simplifying the scanning from the mechanical view point. Although the number of needed NF data is slightly greater than the one required when applying the nonredundant sampling representation on the helix, it results remarkably smaller with respect to that required by the standard cylindrical scanning or by the half-wavelength helicoidal scanning. With respect to the direct helicoidal NF-FF transformation developed in a previous paper and using a prolate ellipsoid to model the AUT, the here proposed one allows generally a further reduction of the NF data and, as a consequence, of the measurement time. As a matter of fact, the rounded cylinder modelling allows one to fit better the shape of a lot of actual antennas by properly setting its geometric parameters. In any case, since there is no practical difference between these two techniques from the computational and accuracy viewpoints, the choice depends only on the modelling which better fits the actual AUT geometry.

\section{CONFLICT OF INTEREST}

The authors confirm that this article content has no conflicts of interest.

\section{ACKNOWLEDGEMENT}

Declared none.

\section{REFERENCES}

[1] R.G. Yaccarino, L.I. Williams, and Y. Rahmat-Samii, "Linear spiral sampling for the bipolar planar antenna measurement technique," IEEE Trans. Antennas Propag., vol. AP-44, pp. 10491051, July 1996.

[2] S. Costanzo, and G. Di Massa, "Far-field reconstruction from phaseless near-field data on a cylindrical helix," J. Electromagn. Waves Appl., vol. 18, pp. 1057-1071, 2004.

[3] O.M. Bucci, C. Gennarelli, G. Riccio, and C. Savarese, "Nonredundant NF-FF transformation with helicoidal scanning," $J$. Electromagn. Waves Appl., vol. 15, pp. 1507-1519, 2001.

[4] F. D'Agostino, C. Gennarelli, G. Riccio, and C. Savarese "Theoretical foundations of near-field-far-field transformations with spiral scannings," Prog. Electromagn. Res., vol. PIER 61, pp.193-214, 2006.

[5] F. D'Agostino, F. Ferrara, C. Gennarelli, R. Guerriero, and M. Migliozzi, "Experimental results validating the near-field to farfield transformation technique with helicoidal scan," Open Electr. Electron. Eng. J., vol. 4, pp. 10-15, 2010.

[6] F. D’Agostino, F. Ferrara, C. Gennarelli, R. Guerriero, and M. Migliozzi, "Near-field - far-field transformation technique with helicoidal scanning for elongated antennas," Prog. Electromagn. Res. B, vol. 4, pp. 249-261, 2008.

[7] F. D’Agostino, F. Ferrara, C. Gennarelli, R. Guerriero, and M Migliozzi, "Laboratory tests assessing the effectiveness of the NF FF transformation with helicoidal scanning for electrically long antennas," Prog. Electromagn. Res., vol. PIER 98, pp. 375-388, 2009.

[8] F. D'Agostino, F. Ferrara, C. Gennarelli, R. Guerriero, and M. Migliozzi, "The unified theory of near-field - far-field transformations with spiral scannings for nonspherical antennas," Prog. Electromagn. Res. B, vol. 14, pp. 449-477, 2009. 
[9] F. D'Agostino, F. Ferrara, J.A. Fordham, C. Gennarelli, R. Guerriero, M. Migliozzi, G. Riccio, and C. Rizzo, "An effective $\mathrm{NF}-\mathrm{FF}$ transformation technique for elongated antennas using a fast helicoidal scan," IEEE Antennas Propag. Mag., vol. 51, pp. 134-141, August 2009.

[10] O.M. Bucci, C. Gennarelli, and C. Savarese, "Representation of electromagnetic fields over arbitrary surfaces by a finite and nonredundant number of samples," IEEE Trans. Antennas Propag., vol. 46, pp. 351-359, March 1998.

[11] O.M. Bucci, and C. Gennarelli, "Application of nonredundant sampling representations of electromagnetic fields to NF-FF transformation techniques," Int. J. Antennas Propag., vol. 2012, p. 319856.

[12] W.M. Leach Jr., and D.T. Paris, "Probe compensated near-field measurements on a cylinder," IEEE Trans. Antennas Propag., vol. AP-21, pp. 435-445, July 1973.

[13] A.D. Yaghjian, "Near-field antenna measurement on a cylindrical surface: a source scattering matrix formulation," NBS Tech. note 696, U.S. Government Printing Office: Washington, 1977.

[14] F. D'Agostino, F. Ferrara, C. Gennarelli, R. Guerriero, and M. Migliozzi, "An innovative direct NF-FF transformation technique with helicoidal scanning," Int. J. Antennas Propag., vol. 2012, p. 912948 .
[15] F. D'Agostino, F. Ferrara, C. Gennarelli, R. Guerriero, and M. Migliozzi, "Evaluation of the far field radiated by long antennas directly from data acquired through a fast helicoidal scanning," Prog. Electromagn. Res. M, vol. 26, pp. 157-171, 2012.

[16] F. D'Agostino, F. Ferrara, C. Gennarelli, R. Guerriero, and M. Migliozzi, "Far-field evaluation directly from helicoidal near-field data," Proc. of European Conference on Antennas \& Propagation (EUCAP), March 2012, paper n. 1569521795.

[17] O.M. Bucci, G. D'Elia, and M.D. Migliore, "Advanced field interpolation from plane-polar samples: experimental verification," IEEE Trans. Antennas Propag., vol. 46, pp. 204-210, February 1998.

[18] O.M. Bucci, C. Gennarelli, G. Riccio, and C. Savarese, "NF-FF transformation with cylindrical scanning: an effective technique for elongated antennas," IEE Proc. Microwaves Antennas Propag., vol. 145, pp. 369-374, October 1998.

[19] F. D'Agostino, F. Ferrara, C. Gennarelli, R. Guerriero, and M. Migliozzi, "On the direct nonredundant near-field - far-field transformation in a cylindrical scanning geometry," IEEE Antennas Propag. Mag., vol. 54, pp. 130-138, February 2012.

[20] A.D. Yaghjian, "Approximate formulas for the far field and gain of open-ended rectangular waveguide," IEEE Trans. Antennas Propag., vol. 34, pp. 378-384, April 1984.

(C) D'Agostino et al.; Licensee Bentham Open.

This is an open access article licensed under the terms of the Creative Commons Attribution Non-Commercial License (http://creativecommons.org/licenses/by$\mathrm{nc} / 3.0 /$ ), which permits unrestricted, non-commercial use, distribution and reproduction in any medium, provided the work is properly cited. 